\title{
Todos têm direito a saúde? Rastreamento de Doenças Crônicas Não Transmissíveis em população sem cobertura de Estratégia Saúde da Família
}

\author{
Is everyone entitled to health? Screening for Chronic Noncommunicable Diseases in a population \\ without coverage under the Family Health Strategy \\ ¿Todos tienen derecho a la salud? Cribado de Enfermedades Crónicas No Transmisibles en una \\ población sin cobertura de la Estrategia Salud de la Familia
}

Recebido: 02/02/2021 | Revisado: 05/02/2021 | Aceito: 02/03/2021 | Publicado: 08/03/2021

\author{
Carla Craus Móvio \\ ORCID: https://orcid.org/0000-0002-3619-3583 \\ Universidade Cesumar Unicesumar, Brasil \\ E-mail: carlacraus15@gmail.com \\ Fernanda Luchetti Fernandes \\ ORCID: https://orcid.org/0000-0002-3778-8335 \\ Universidade Cesumar Unicesumar, Brasil \\ E-mail: fernandaluchetti26@gmail.com \\ Maria Dalva Barros Carvalho \\ ORCID: https://orcid.org/0000-0002-1377-3331 \\ Centro Universitário Ingá Uningá, Brasil \\ E-mail: dbcarvalho@gmail.com \\ Silvia Veridiana Zamparoni Victorino \\ ORCID: https://orcid.org/0000-0003-4322-3901 \\ Universidade Estadual de Maringá, Brasil \\ E-mail: veridiana.victorino@gmail.com
}

\begin{abstract}
Resumo
Objetivo: Rastrear as Doenças Crônicas Não Transmissíveis como Diabetes Mellitus, Hipertensão Arterial e Obesidade em população sem cobertura de Estratégia Saúde da Família. Método: Pesquisa observacional transversal, de abordagem quantitativa com dados primários. A amostra foi composta por 62 indivíduos maiores de 18 anos, moradores de um bairro localizado na zona rural de Sarandi-PR. Os dados foram coletados através da aferição da pressão arterial, verificação da glicemia capilar e levantamento das medidas antropométricas. Os dados foram tabulados com a utilização de planilhas do Microsoft Excel e após isso realizou-se uma análise descritiva para caracterizar os indivíduos, utilizando a frequência absoluta e a porcentagem para as variáveis categóricas. Resultados: Dos sujeitos da pesquisa, 74,2\% apresentaram IMC alterado, sendo 76,0\% com companheiro fixo e $24,0 \%$ sem. Quanto ao risco cardiovascular 59 (96,7\%) apresentaram risco elevado. A proporção entre homens e mulheres quanto aos riscos cardiovasculares foi de 1,95:2. 66,1\% dos morados apresentaram alteração da pressão e 29,0\% apresentaram alteração na glicemia capilar tanto em jejum como pós-prandial. Conclusão: Conclui-se que a população investigada apresenta maior prevalência de DCNTs com relação a população geral brasileira. Apontando uma necessidade de intervenção focada nos comportamentos modificáveis, que pode ser realizada pela Atenção Primária em Saúde por meio da Estratégia Saúde da Família.
\end{abstract}

Palavras-chave: Doenças crônicas; Obesidade; Hipertensão; Diabetes; Estratégia saúde da família.

\begin{abstract}
Objective: To track Chronic Noncommunicable Diseases such as Diabetes Mellitus, Arterial Hypertension and Obesity in a population not covered by the Family Health Strategy. Method: Cross-sectional observational research, with a quantitative approach with primary data. The sample consisted of 62 individuals over 18 years of age, living in a neighborhood located in the rural area of Sarandi-PR. Data were collected by measuring blood pressure, checking capillary blood glucose and surveying anthropometric measurements. The data was tabulated using Microsoft Excel spreadsheets and afterwards a descriptive analysis was performed to characterize the individuals, using the absolute frequency and percentage for categorical variables. Results: Of the research subjects, $74.2 \%$ had an altered BMI, $76.0 \%$ with a steady partner and $24.0 \%$ without. As for cardiovascular risk, $59(96.7 \%)$ were at high risk. The proportion between men and women regarding cardiovascular risks was $1.95: 2.66 .1 \%$ of the residents had changes in blood pressure and $29.0 \%$ had changes in capillary blood glucose, both fasting and postprandial. Conclusion: It is concluded that the investigated population has a higher prevalence of NCDs compared to the general Brazilian
\end{abstract}


population. Pointing out a need for intervention focused on modifiable behaviors, which can be performed by Primary Health Care through the Family Health Strategy.

Keywords: Chronic diseases; Obesity; Hypertension; Diabetes; Family health strategy.

\begin{abstract}
Resumen
Objetivo: Realizar el seguimiento de Enfermedades Crónicas No Transmisibles como Diabetes Mellitus, Hipertensión Arterial y Obesidad en una población no cubierta por la Estrategia de Salud de la Familia. Método: Investigación observacional transversal, con enfoque cuantitativo con datos primarios. La muestra estuvo conformada por 62 individuos mayores de 18 años, residentes en un barrio ubicado en la zona rural de Sarandi-PR. Los datos se recopilaron midiendo la presión arterial, controlando la glucosa en sangre capilar y midiendo las mediciones antropométricas. Los datos se tabularon mediante planillas de Microsoft Excel y posteriormente se realizó un análisis descriptivo para caracterizar a los individuos, utilizando la frecuencia absoluta y el porcentaje para las variables categóricas. Resultados: De los sujetos de investigación, el 74,2\% tenía un IMC alterado, el 76,0\% con una pareja estable y el 24,0\% sin él. En cuanto al riesgo cardiovascular, 59 (96,7\%) tenían alto riesgo. La proporción entre hombres y mujeres con respecto a los riesgos cardiovasculares fue de 1,95: 2. El 66,1\% de los residentes presentó cambios en la presión arterial y el 29,0\% tuvo cambios en la glucemia capilar, tanto en ayunas como posprandial. Conclusión: Se concluye que la población investigada tiene una mayor prevalencia de ECNT en comparación con la población brasileña en general. Señalar la necesidad de una intervención centrada en conductas modificables, que puede ser realizada por la Atención Primaria de Salud a través de la Estrategia de Salud de la Familia.
\end{abstract}

Palabras clave: Enfermedades crónicas; Obesidad; Hipertensión; Diabetes; Estrategia de salud familiar.

\title{
1. Introdução
}

Em tempos modernos as Doenças Crônicas não Transmissíveis (DCNTs) são consideradas umas das maiores vilãs da saúde, devido sua alta prevalência e morbimortalidade. No mundo, segundo a Organização Mundial da Saúde (OMS) (2019), em 2016 as DCNTs foram responsáveis pela morte de 41 milhões de pessoas. No Brasil, por ser um país de grande extensão territorial com diversidade cultural e econômica, existe uma variação dos índices de prevalência das DCNTs, sendo que, de acordo com o Ministério da Saúde (MS) (2020), a prevalência de obesidade varia entre 15,4\% a 23,4\%, a da Diabetes Mellitus (DM) de 4,6\% a 8,6\% e a da Hipertensão Arterial Sistêmica (HAS) varia de 16,9\% a 28,5\%.

As DCNTs estão diretamente ligadas ao estilo de vida atual, em que uma rotina agitada acarreta em estresse, e uma grande oferta de tecnologia para o trabalho e lazer favorece o sedentarismo. Além de uma alimentação nada sau dável com alto consumo de alimentos processados e calóricos ser cada vez mais procurada em meio a sociedade (Reis et al., 2020).

Com a transição demográfica e aumento do número de pessoas idosas, ocorre a elevação da prevalência de DCNTs e do monitoramento das mesmas. A exemplo da hipertensão arterial, em que a falta de vigilância está relacionada à idade e quantidade de medicamentos, sendo que quanto maior a idade e o número de medicamentos, menor o controle da mesma (Pinhatti et al., 2019).

A literatura comprova que existem fatores de risco ocupacionais que muito tem contribuído para carga global de DCNTs, como fatores ergonômicos e ruídos no ambiente de trabalho (Assunção \& França, 2020), assim a população tem desenvolvido tais comorbidades mais precocemente, sendo um grande problema para a saúde pública e privada de nosso país. Pessoas portadoras de DCNTs e com maior número de comorbidades são as que mais utilizam o sistema de saúde público e privado de saúde (Malta et al., 2020).

A Estratégia Saúde da Família (ESF) apresenta um grande potencial para o manejo da prevenção das DCNTs e o seu controle, evitando assim um número cada vez maior de internações. (Pereira, Santos \& Uherara, 2020). Devido a isso, o Ministério da Saúde, nas últimas décadas, tem investido muito nesta estratégia. Todavia, ainda é possível encontrar, em território nacional, locais que não contam com a cobertura da ESF.

Desse modo, o rastreamento dessas doenças crônicas, especialmente em populações desassistidas pela ESF se faz necessário para a início do desenvolvimento de projetos que possam modificar esta realidade. 
O objetivo desta pesquisa foi rastrear as Doenças Crônicas Não transmissíveis como DM, HAS e obesidade em população sem cobertura de Estratégia Saúde da Família.

\section{Metodologia}

Pesquisa observacional transversal de abordagem quantitativa com dados primários.

Realizada no bairro rural Vale Azul, no município de Sarandi, localizado na região norte do estado do Paraná, o qual conta com 97.803 habitantes, segundo o Instituto Brasileiro de Geografia e Estatística (IGBE) (2021). Neste bairro, não há informações oficiais da quantidade de residentes. Os moradores referem que tem em torno de 500 residências com aproximadamente 2000 habitantes. Neste local não há infraestrutura urbana, com carência de unidades básicas de saúde, áreas de lazer, entre outros. Há a presença de apenas uma unidade de saúde com estrutura física e humana mínima, não caracterizada como UBS, a qual oferece somente serviços médicos e odontológicos simples uma vez por semana, realizando exclusivamente procedimentos básicos e não invasivos.

A coleta de dados foi realizada no período de dezembro de 2019 a março de 2020 pelos próprios pesquisadores através de visita domiciliar. Os sujeitos alvos da pesquisa foram moradores acima de 18 anos.

Os dados foram coletados através da aferição da pressão arterial (PA), verificação da glicemia capilar (GC) e levantamento das medidas antropométricas. Para a aferição da PA foram utilizados: esfigmomanômetros Welch Allyn calibrados e estetoscópio Littman Classic III. A técnica utilizada para essa aferição foi a recomendada pela $7^{\circ}$ Diretriz Brasileira de Hipertensão Arterial da Sociedade Brasileira de Cardiologia (SBC) (2016). Os valores de parâmetro foram os preconizados pela mesma diretriz.

Para a verificação da GC foi utilizado o glicosímetro ACCU-CHECK Active. A execução desta técnica seguiu o procedimento operacional padrão (POP) da Universidade Federal de Santa Catarina (Xavier \& Prudente, 2016).

De acordo com a Sociedade Brasileira de Diabetes (SBD) (2019), a GC é um método utilizado para detectar flutuações da glicemia ao longo do dia. Portanto, os resultados, se muito alterados, são parâmetros para orientação de auxílio médico. Assim, a utilização deste método de acordo com as Diretrizes da Sociedade Brasileira de Diabetes é uma importante estratégia que pode colaborar para subsidiar ações de educação em saúde, considerando que a comunidade alvo da pesquisa tem restrito acesso às ações de saúde pública.

Os parâmetros de GC utilizados em jejum (até $100 \mathrm{mg}$ ) e pós-prandial de 2 horas (até $140 \mathrm{mg}$ ) foram os mesmos preconizados pela Sociedade Brasileira de Diabetes (SBD, 2019) como critérios glicêmicos para rastreio de DM.

Para a verificação das medidas antropométricas foram utilizadas a fita métrica, balança eletrônica portátil Balmack de $200 \mathrm{Kg}$ e calculadora. A coleta das medidas antropométricas seguiu as recomendações do Ministério da Saúde (MS, 2011).

Os dados foram tabulados com a utilização de planilhas do Microsoft Excel e após realizou-se uma análise descritiva para caracterizar os indivíduos, utilizando a frequência absoluta e a porcentagem para as variáveis categóricas. Para averiguação de associação entre variáveis foi utilizado o teste, $\chi^{2}$. Todas as análises foram realizadas com o auxílio do ambiente estatístico R ( $R$ Development Core Team), versão 3.5.

A pesquisa foi submetida e aprovada pelo Comitê de Ética em pesquisa com seres humanos da Unicesumar, parecer $\mathrm{n}^{\circ}$ 3.651.684. Os participantes receberam 2 duas vias e assinaram o Termo de Consentimento Livre Esclarecido. 


\section{Resultados}

A amostra foi composta por 62 pessoas, destas 62,9\% eram mulheres, $77 \%$ apresentavam menos de 8 anos de escolaridade, $60,7 \%$ eram católicos e 51,6\% eram brancos (Tabela 1).

Tabela 1: Distribuição de frequências e porcentagem de variáveis sócio demográficas. Sarandi-PR.

\begin{tabular}{|c|c|c|}
\hline & Frequência & Porcentagem \\
\hline \multicolumn{3}{|l|}{ Sexo } \\
\hline Feminino & 39 & $62,9 \%$ \\
\hline Masculino & 23 & $37,1 \%$ \\
\hline \multicolumn{3}{|l|}{ Raça/cor } \\
\hline Branco & 32 & $51,6 \%$ \\
\hline Negro & 6 & $9,7 \%$ \\
\hline Pardo & 24 & $38,7 \%$ \\
\hline \multicolumn{3}{|l|}{ Escolaridade } \\
\hline Menos de 8 & 48 & $77,4 \%$ \\
\hline Mais de 8 & 14 & $22,6 \%$ \\
\hline \multicolumn{3}{|l|}{ Religião } \\
\hline Católica & 34 & $60,7 \%$ \\
\hline Evangélica & 20 & $35,7 \%$ \\
\hline Espírita & 1 & $1,8 \%$ \\
\hline Testemunha de Jeová & 1 & $1,8 \%$ \\
\hline
\end{tabular}

Fonte: Autores.

Na distribuição etária (Figura 1), houve uma grande amplitude na idade indicando assimetria, com uma frequência expressiva de moradores entre 50 e 70 anos. Observou-se uma relação entre as variáveis, porém não linear, visto que a idade mais comum foi cerca de 56 anos (moda), a média de idade foi de 60,2 anos (DP \pm 13,6) e mediana de 61,0 anos. A idade mínima foi de 28 anos e a máxima de 85 anos. 
Research, Society and Development, v. 10, n. 3, e10810312581, 2021

(CC BY 4.0) | ISSN 2525-3409 | DOI: http://dx.doi.org/10.33448/rsd-v10i3.12581

Figura 1: Histograma de distribuição etária da amostra. Sarandi-PR, 2020.

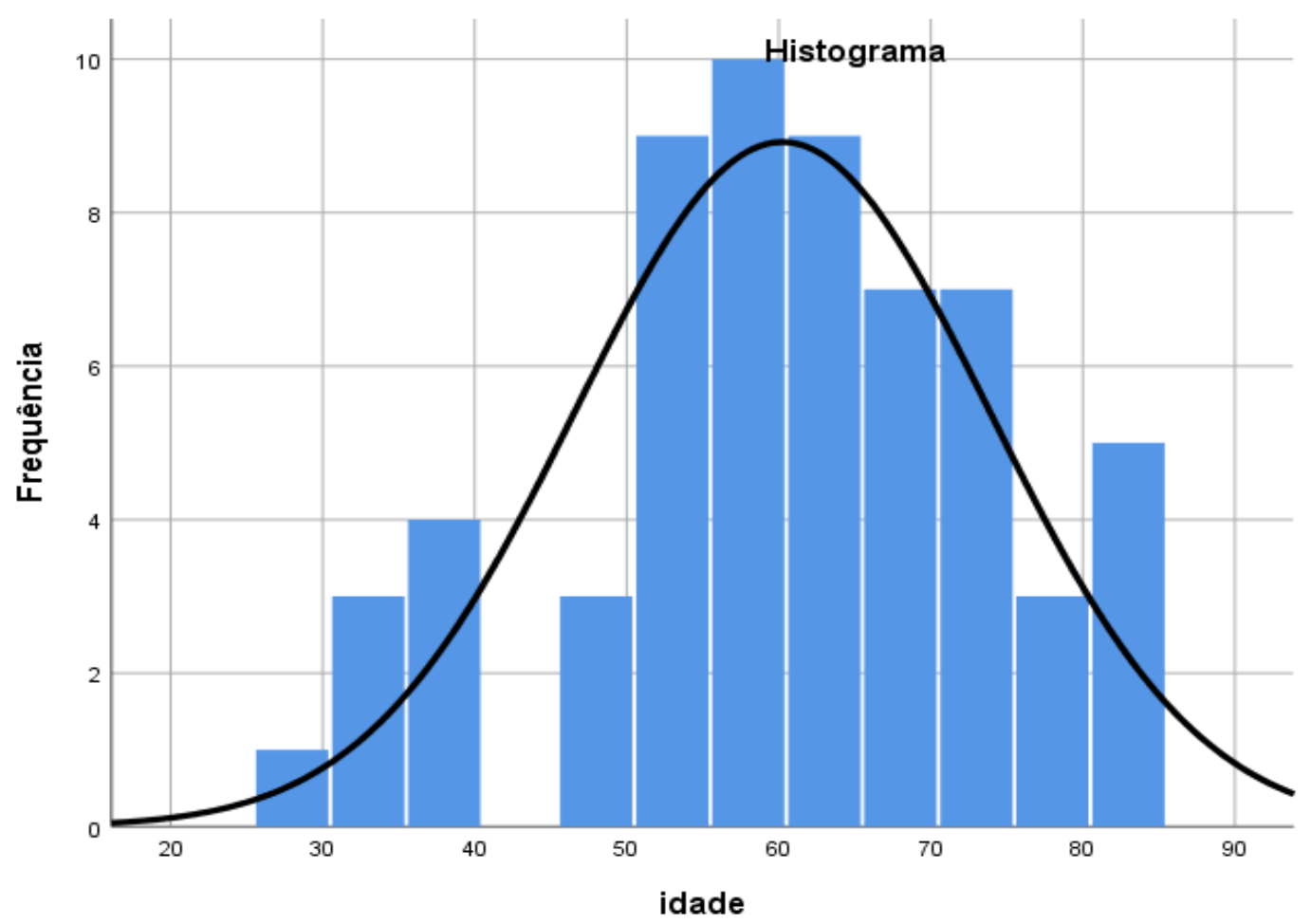

Fonte: Autores.

As próximas Tabelas (2 e 3) demonstram resultados estatisticamente relevantes, em que há uma relação significativa entre sexo e estado civil com o ganho de peso. 
Tabela 2: Análise descritiva e teste de associação entre as variáveis IMC, GC e PA em relação ao sexo dos residentes do Bairro Vale Azul, Sarandi-PR.

\begin{tabular}{|c|c|c|c|}
\hline Variável & FEMININO & MASCULINO & P valor \\
\hline \multicolumn{4}{|l|}{ IMC } \\
\hline Normal & $8(50,0 \%)$ & $8 \quad(50,0 \%)$ & $\leq 0,05$ \\
\hline Sobrepeso & $12(54,5 \%)$ & $10(45,5 \%)$ & \\
\hline Obesidade grau 1 & $12(70,6 \%)$ & $5(29,4 \%)$ & \\
\hline Obesidade grau 2 & $4(100,0 \%)$ & $0(0,0 \%)$ & \\
\hline Obesidade grau 3 & $3(100,0 \%)$ & $0(0,0 \%)$ & \\
\hline TOTAL & $39(62,9 \%)$ & $23(37,1 \%)$ & \\
\hline GC & & & 0,98 \\
\hline Normal & $27(61,4 \%)$ & $17(38,6 \%)$ & \\
\hline Pré DM & $8(72,7 \%)$ & $3(27,3 \%)$ & \\
\hline $\mathrm{DM}$ & $4(57,1 \%)$ & $3(42,9 \%)$ & \\
\hline TOTAL & $39(62,9 \%)$ & $23(37,1 \%)$ & \\
\hline PA & & & 0,19 \\
\hline Normal & $12(57,1 \%)$ & $9(42,9 \%)$ & \\
\hline Pré-Hipertensão & $10(58,8 \%)$ & $7(41,2 \%)$ & \\
\hline HAS 1 & $10(66,7 \%)$ & $5(33,3 \%)$ & \\
\hline HAS 2 & $3(60,0 \%)$ & $2(40,0 \%)$ & \\
\hline HAS 3 & $4(100,0 \%)$ & $0(0,0 \%)$ & \\
\hline TOTAL & $39(62,9 \%)$ & $23(37,1 \%)$ & \\
\hline
\end{tabular}

Fonte: Autores.

A Tabela 2 evidencia maior relação de sobrepeso e obesidade entre o sexo feminino. Dentre os residentes, 74,2\% apresentaram IMC alterado, destes 67,4\% eram mulheres e 32,6\%, homens. Os homens apresentaram sobrepeso (45,5\%) e obesidade grau $1(29,4 \%)$ e as mulheres apresentaram sobrepeso (54,5\%), obesidade grau 1 (70,6\%), obesidade grau 2 $(100,0 \%)$ e obesidade grau $3(100,0 \%)$. No geral 38,7\% dos sujeitos apresentaram algum grau de obesidade.

Os valores da relação cintura/quadril são estratificados por sexo e idade em baixo, moderado, alto e muito alto risco. Dos 61 sujeitos (perda de 1) analisados, 59 (96,7\%) apresentaram risco cardiovascular, $39(66,1 \%)$ mulheres e 20 (33,9\%) homens. A proporção entre homens e mulheres quanto aos riscos cardiovasculares foi de 1, 95:2. Dois homens apresentaram risco moderado e três mulheres risco muito alto para a faixa etária de 30 a 39 anos. Na faixa etária de 40 a 49 anos, 4 mulheres apresentaram risco muito alto e na faixa de 50 a 59 anos 4 homens apresentaram risco moderado, 1 risco muito alto e 1 alto risco. Já entre os homens com mais de 60 anos 1 se classificou como risco baixo, 3 como risco moderado, 5 como alto risco e 3 como muito alto risco. Entre as mulheres 18 apresentaram risco muito alto, 4 alto risco e 1 risco moderado.

A glicemia capilar foi coletada em jejum $(34$ - 54,8\%) e pós-prandial (28 -45,3\%). Dos 62 sujeitos avaliados por GC, $18(29,0 \%)$ apresentaram alteração tanto em jejum como pós-prandial. As mulheres foram em maior número 12 (66,6\%). Entre 
os resultados mais elevados, acima de 200 (5), as mulheres eram maioria (3). As 4 mulheres na faixa etária de 30 a 40 anos que apresentaram resultados alterados, 3 estavam com sobrepeso, uma com obesidade e todas tinham risco cardiovascular alto e/ou muito alto.

Entre os 62 moradores avaliados através da pressão arterial, 66,1\% apresentaram alteração da PA, sendo 27 (65,8\%) mulheres e 14 (34,2\%) homens. Dentre os residentes com HAS estágio 1, $10(66,7 \%)$ eram do sexo feminino e 5 (33,3\%) do sexo masculino. Além disso, dos residentes com HAS estágio 2 (5), três eram mulheres (60\%), e 100\% dos moradores com HAS estágio 3 eram do sexo feminino.

Tabela 3: Análise descritiva e teste de associação entre variáveis IMC e PA com e sem companheiro dos residentes do Bairro Vale Azul, Sarandi-PR.

\begin{tabular}{|c|c|c|c|}
\hline Variáveis & Com companheiro & Sem companheiro & $P$ valor \\
\hline IMC & & & $\leq 0,05$ \\
\hline Normal & $6(37,5 \%)$ & $10(62,5 \%)$ & \\
\hline Sobrepeso & $16(72,7 \%)$ & $6(27,3 \%)$ & \\
\hline Obesidade grau 1 & $14(82,4 \%)$ & $3(17,6 \%)$ & \\
\hline Obesidade grau 2 & $3(75,0 \%)$ & $1(25,0 \%)$ & \\
\hline Obesidade grau 3 & $2(66,7 \%)$ & $1(4,8 \%)$ & \\
\hline TOTAL & $41(66,12 \%)$ & $21(33,88 \%)$ & \\
\hline $\mathbf{P A}$ & & & 0,14 \\
\hline Normal & $14(66,7 \%)$ & $7(33,3 \%)$ & \\
\hline Limítrofe & $14(82,4 \%)$ & $3(17,6 \%)$ & \\
\hline HAS 1 & $9(60,0 \%)$ & $6(40,0 \%)$ & \\
\hline HAS 2 & $3(60,0 \%)$ & $2(40,0 \%)$ & \\
\hline HAS 3 & $1(25,0 \%)$ & $3(75,0 \%)$ & \\
\hline TOTAL & $41(66,12 \%)$ & $21(33,88 \%)$ & \\
\hline
\end{tabular}

Fonte: Autores.

Na Tabela 3 destaca-se a relação significativa entre IMC alterado e residentes com companheiro fixo. Como resultado, 74,2\% dos moradores apresentaram algum grau de obesidade e/ou sobrepeso, sendo 76,0\% com companheiro fixo e $24,0 \%$ sem companheiro fixo 


\section{Discussão}

Neste estudo, as mulheres mostraram-se em maior número e mais solícitas em relação à pesquisa e responsivas às perguntas realizadas para o levantamento de dados. Diferentemente, os homens, provavelmente influenciados por fatores culturais relacionados a masculinidade, crescem com o pressuposto julgamento que necessitam supervisionar seus gestos, atitudes emocionais e o próprio corpo, repercutindo uma ideia negativa sobre a busca do cuidado com a saúde e gerando o pensamento de que ao buscar auxílio médico entrará em conflito com sua força e virilidade masculina. (Botton, Cúnico \& Strey, 2017; Sabóia et al., 2016).

$\mathrm{Na}$ presente pesquisa, foi identificado que 74,2\% da amostra encontrava-se com o IMC alterado, apresentando sobrepeso ou algum grau de obesidade. Esse achado se aproximou da prevalência encontrada em estudo de uma população urbana carente do nordeste brasileiro, a qual foi de 70,3\% (Melo et al., 2020). Em relação a obesidade geral, aproximadamente $40 \%$ dos sujeitos da amostra apresentaram algum grau de obesidade. Este resultado quando comparado com dados da Pesquisa Nacional de Saúde é alarmante, visto que, os dados nacionais apontam uma prevalência de obesidade geral de 20,8\% (MartinsSilva, Vaz, Mola, Assunção \& Rodrigues, 2019).

Também foi constatado, neste estudo, uma relação estatisticamente relevante entre indivíduos com companheiro fixo e IMC elevado. De acordo com a Associação Brasileira para o Estudo da Obesidade e Síndrome Metabólica (ABESO) (2016), em uma união estável, a alimentação torna-se mais calórica, e o gasto energético é reduzido. Ademais, Teachman (2016) aponta que pessoas com companheiro fixo, pelo fato de desfrutarem de uma companhia na refeição se alimentam mais regularmente levando ao ganho de peso. Além disso, pelo motivo de já apresentarem parceiros e não estarem a procura de alguém, não se atentam ao ganho de peso corporal.

Sabe-se que a obesidade tem aumentado nas últimas décadas, principalmente no sexo feminino. (Araújo, Aguiar \& Fonseca, 2019). No presente estudo, verificou-se maior prevalência de obesidade e risco cardiovascular entre as mulheres, corroborando com o encontrado no estudo de coorte realizado em capitais brasileiras. (Martins-Silva et al., 2019).

Nesta pesquisa, aproximadamente $97 \%$ dos indivíduos apresentaram risco cardiovascular elevado, resultado este maior do que os descritos na literatura, que em idosos ficou em 46,8\% para risco moderado e 26,0\% para risco elevado, sendo que o sexo feminino e a idade avançada influenciaram negativamente esses valores, como evidenciado pelo trabalho de Costa et al. (2021).

A composição corporal difere entre homens e mulheres. As mulheres proporcionalmente apresentam maior concentração de gordura corporal localizada em extremidade inferior (pear shape) a qual está associada a um perfil de risco cardiometabólico mais favorável, como evidenciado por Schorr et al. (2018). Contudo, o ato de envelhecer estabelece mudanças fisiológicas na composição corporal, como alterações hormonais, menor disposição para a prática de atividades físicas, diminuição da taxa metabólica basal e do metabolismo (Sabóia et al., 2016; Schorr et al., 2018). Além disso, a obesidade abdominal está relacionada ao tabagismo, consumo regular de bebida alcóolica, sedentarismo, idade superior a 40 anos e viver com companheiro, sendo a prevalência mais elevada no sexo feminino. A idade entre 30-59 anos e viver com companheiro são considerados fatores de risco para ambos os sexos, já possuir maior escolaridade é considerado fator de proteção. (Sabóia et al., 2016).

Nesta pesquisa, a maior prevalência de hipertensão arterial sistêmica se deu entre o sexo feminino, corroborando com dados da Pesquisa Nacional de Saúde (PNS) (Oliveira, 2018). A prevalência de HAS é maior em regiões com baixas condições socioeconômicas, por estas serem mais expostas à fatores de risco e apresentarem menor acesso aos serviços de saúde. Além disso, baixa escolaridade, sexo feminino, sedentarismo e excesso de peso são considerados importantes fatores para o desenvolvimento desta DCNT (Bernal et al., 2020; Fiório, Cesar, Alves \& Goldbaum, 2020). 
Além disso, as medidas de GC encontradas foram mais alteradas no sexo feminino. Isso pode ser explicado pelo fato de as mulheres possuírem mais fatores de risco, como sedentarismo e hábitos alimentares não saudáveis, com dieta inadequada pobre em fibras e vitaminas. Além disso, as modificações que ocorrem no organismo durante a gravidez podem contribuir para o desenvolvimento de diabetes, como a diabetes mellitus gestacional (Araújo, 2018).

A pesquisa comprovou que existe uma alta prevalência de DCNTs, acarretando grandes limitações para o indivíduo e atingindo diretamente sua qualidade de vida, além de ser um fator de extrema importância para o sistema público, uma vez que sobrecarrega os serviços de saúde, dispendendo muitos gastos com estas comorbidades. Em 2018, o custo atribuído para obesidade, HAS e DM foi de R \$ 1,42 bilhão, isto significa 41\% dos custos totais com saúde (Nilson, Andrade, Brito \& Oliveira, 2018).

Neste estudo, houve, como limitante, o número pequeno de entrevistados. Todavia, ainda assim, foram estabelecidas associações estatísticas significativas. Os resultados, deste modo, indicam caminhos para o desenvolvimento de atividades em saúde voltadas para a diminuição dos fatores de risco desta população.

\section{Conclusão}

Conclui-se que a população investigada apresenta maior prevalência de DCNTs em relação a população geral brasileira, apontando uma necessidade de intervenção focada nos comportamentos modificáveis. Essa intervenção pode ser realizada pela Atenção Primária em Saúde, por meio da Estratégia Saúde da Família.

Sugere-se que seja implantada uma equipe da Estratégia Saúde da Família nesta comunidade e que seja feito um programa de educação em saúde, o qual deverá privilegiar estratégias que atinjam o indivíduo de forma que as ações façam significado para ele. Só assim poderá haver mudança de comportamento a longo prazo, de maneira voluntária, com sentido para o indivíduo. Uma intervenção planejada e constante para diminuir os riscos a que as pessoas estão expostas é necessária.

Novas investigações devem ser feitas nesta comunidade, focando as crianças, sua condição de saúde e influência da instituição escolar no desenvolvimento de comportamento saudável dos estudantes.

\section{Referências}

Araújo, F. G. (2018). Tendência da prevalência de sobrepeso, obesidade, diabetes e hipertensão em mulheres brasileiras em idade reprodutiva, Vigitel 2008-2015. Dissertação de mestrado, Universidade Federal de Minas Gerais, Belo Horizonte, MG, Brasil.

Araújo, T. P. de, Aguiar, O. B. de, \& Fonseca, M. de J. M. da (2019). Incidência de ganho de peso em trabalhadores de um hospital: análise de sobrevivência. Ciência \& Saúde Coletiva, 24(10), 3847-3856. https://doi.org/10.1590/1413-812320182410.03412018

Associação Brasileira para o Estudo da Obesidade e Síndrome Metabólica (2016). Diretrizes Brasileiras de Obesidade. https://abeso.org.br/wpcontent/uploads/2019/12/Diretrizes-Download-Diretrizes-Brasileiras-deObesidade-2016.pdf.

Assunção, A. A., \& França, E. B. (2020). Anos de vida perdidos por DCNT atribuídos aos riscos obionais no Brasil: estudo GBD 2016. Revista de Saúde Pública, 54, 28. https://doi.org/10.11606/s1518-8787.2020054001257

Bernal, R. T. I., Felisbino-Mendes, M. S., Carvalho, Q. Hapuque de, Pell, J., Dundas, R., Leyland, A., Barreto, M. L., \& Malta, D. C. (2019). Indicadores de doenças crônicas não transmissíveis em mulheres com idade reprodutiva, beneficiárias e não beneficiárias do Programa Bolsa Família. Revista Brasileira de Epidemiologia, 22(2), E190012. https://dx.doi.org/10.1590/1980-549720190012.supl.2

Botton, A., Cúnico, S. D., \& Strey, M. N. (2017). Diferenças de gênero no acesso aos serviços de saúde: problematizações necessárias. Mudanças - Psicologia da Saúde, 25(1): 67-72. https://doi.org/10.15603/2176-1019/mud.v25n1p67-72

Costa, M. V. G. da, Lima, L. R. de, Silva, I. C. R. da, Rehem, T. C. M. S. B., Funghetto, S. S., \& Stival, M. M. (2021). Risco cardiovascular aumentado e o papel da síndrome metabólica em idosos hipertensos. Escola Anna Nery, 25(1), e20200055. https://dx.doi.org/10.1590/2177-9465-ean-2020-0055

Fiório, C. E., Cesar, C. L. G., Alves, M. C. G. P., \& Goldbaum, M. (2020). Prevalência de hipertensão arterial em adultos no município de São Paulo e fatores associados. Revista Brasileira de Epidemiologia, 23, e200052. https://doi.org/10.1590/1980-549720200052 
Malta, D. C., Bernal, R. T. I., Carvalho, Q. H. de, Pell, J. P., Dundas, R., Leyland, A., Vasconcelos, L. L. C. de, Cardoso, L. S. de M., Stopa, S. R., \& Barreto, M. L. (2020). Mulheres e avaliação das desigualdades na distribuição de fatores de risco de doenças crônicas, Vigitel 2016-2017. Revista Brasileira de Epidemiologia, 23, e200058.

Instituto Brasileiro de Geografia e Estatística. (2021). Cidades. https://cidades.ibge.gov.br.

Martins-Silva, T., Vaz, J. dos S., Mola, C. L. de, Assunção, M.C. F., \& Tovo-Rodrigues, L. (2019). Prevalências de obesidade em zonas rurais e urbanas no Brasil: Pesquisa Nacional de Saúde, 2013. Revista Brasileira de Epidemiologia, 22, e190049. https://doi.org/10.1590/1980-549720190049

Melo, S. P. da S. de C., Cesse, E. A. P., Lira, P. I. C. de, Ferreira, L. C. C. do N., Rissin, A., \& Batista, M., F. (2020). Sobrepeso, obesidade e fatores associados aos adultos em uma área urbana carente do Nordeste Brasileiro. Revista Brasileira de Epidemiologia, 23, e200036. https://doi.org/10.1590/1980549720200036

Ministério da Saúde (2011). Orientações para a coleta e análise de dados antropométricos em serviços de saúde. https://bvsms.saude.gov.br/bvs/publicacoes/orientacoes_coleta_analise_dados_antropometricos.pdf.

Ministério da Saúde (2020). Vigitel Brasil 2019: Vigilância de fatores de risco e proteção para doenças crônicas por inquérito telefônico https://portalarquivos.saude.gov.br/images/pdf/2020/April/27/vigitel-brasil-2019-vigilancia fatores-risco.pdf.

Nilson, E. A. F., Andrade, R. C. S., Brito, D. A. de \& Oliveira, M. L. de (2018). Custos atribuíveis a obesidade, hipertensão e diabetes no Sistema Único de Saúde, Brasil. Rev Panam Salud Publica. 44:e32. https://doi.org/10.26633/RPSP.2020.32

Oliveira, I. M. (2018). Hipertensão arterial sistêmica em idosos de São Paulo e fatores associados ao diagnóstico, não diagnóstico e controle: Estudo SABE. Dissertação de mestrado, Universidade de São Paulo, São Paulo, SP, Brasil.

Pereira, H. N., Santos, R. I. de O., \& Uehara, S. C. S. A. (2020). Efeito da Estratégia Saúde da Família na redução de internações por doenças crônicas não transmissíveis. Revista de enfermagem UERJ, 28:e49931.

Pinhati, R. R., Tavares, P. L., Marsicano, E. de O., Fernandes, N. de S., Colugnati, F. A. B., Bastos, M. G., Paula, R.B., \& Sanders-Pinheiro, H. (2019). Baixo letramento em saúde em pacientes idosos com pressão arterial não controlada em nível secundário de atenção à saúde. 45(1):13-21. 10.34019/1982-8047

Reis, B. de S. M.T., Machado, I. E., Freitas, M. I. de F., Jorge, A. de O., Silva, A. G., \& Malta, D. C. (2020). Tendência temporal da prevalência dos fatores de risco e de proteção para doenças crônicas não transmissíveis em belo horizonte, MG. Reme: Revista Mineira de Enfermagem,24, e1307. https://dx.doi.org/10.5935/1415-2762.20200044

Sabóia, R. S., Araújo, A. P., Barbosa, J. M. A., Galvão, C. E. P., Cruvel, J. M. S., \& Ferreira, S. C. do N. (2016). Obesidade abdominal e fatores associados em adultos atendidos em uma clínica escola. Revista Brasileira Promoção da Saúde, Fortaleza, 29(2): 259-267.

Schorr, M., Dichtel, L. E., Gerweck, A. V., Valera, R. D., Torriani, M., Miller, K. K., \& Bredella, M. A. (2018). Sex differences in body composition and association with cardiometabolic risk. Biol Sex Differ. 2018 Jun 27;9(1):28. doi: 10.1186/s13293-018-0189-3.

Sociedade Brasileira de Cardiologia (2016). VII Diretriz Brasileira de Hipertensão Arterial. Arquivos Brasileiros de Cardiologia http://publicacoes.cardiol.br/2014/diretrizes/2016/05_HIPERTENSAO_ARTERIAL.pdf.

Sociedade Brasileira de Diabetes (2019). Diretrizes da Sociedade Brasileira de Diabetes 2019-2020. https://www.diabetes.org.br/pr ofissionais/images/DIRETRIZES-COMPLETA-2019-2020.pdf.

Teachman, J. (2016, January). Body Weight, Marital Status, and Changes in Marital Status. Journal of Family Issues, 37(1): 74-96.

World Health Organization (2019). World health statistics 2019. https://apps.who.int/iris/bitstream/handle/10665/324835/97892415657 07eng.pdf? sequence $=9 \&$ isAllowed $=\mathrm{y}$.

Xavier, L. B., Prudente, A. B. (2016). Procedimento Operacional Padrão: Verificação de glicemia capilar. Núcleo de Educação Permanente em Enfermagem (NEPEn), HU-UFSC. http://www.hu.ufsc.br/documentos/pop/enfermagem/assistenciais/REGULACAO_HORMONAL/VERIFICACAO_GLICEMIA.pdf. 